\title{
The statistics of critical points of Gaussian fields on large-dimensional spaces
}

\author{
Alan J. Bray ${ }^{1}$ and David S. Dean ${ }^{2}$ \\ 1 School of Physics and Astronomy, University of Manchester, Manchester, M13 9Pl, UK \\ ${ }^{2}$ Laboratoire de Physique Théorique (UMR 5152 du CNRS), \\ Université Paul Sabatier, 118, route de Narbonne, 31062 Toulouse Cedex 4, France
}

(Dated: 31 October 2006)

\begin{abstract}
We calculate the average number of critical points of a Gaussian field on a high-dimensional space as a function of their energy and their index. Our results give a complete picture of the organization of critical points and are of relevance to glassy and disordered systems and landscape scenarios coming from the anthropic approach to string theory.

PACS numbers: 02.50.-r, 12.40.Ee, 78.55.Q
\end{abstract}

The statistics of random fields have been studied in detail in the mathematics and physics literature [1]. Gaussian random fields are of particular importance, as they can be argued to occur spontaneously in systems with a large number of degrees of freedom by appealing to the central limit theorem. Recently string theorists have postulated that the effective theory arising from string theory is highly complex leading to a string landscape with a huge number of possible minima, each of which could describe a potential universe [2], thus a statistical study of this complex landscape may well be necessary. Gaussian fields arise generically in the study of classical complex systems such as spin glasses, optimization problems and protein folding [3]. In many of these systems, jamming or the onset of a glass transition occurs due to the presence of a large number of metastable states or local minima in the energy landscape. High-dimensional complex potentials also occur as the potential energy of interacting particle systems which exhibit a structural glass transition (no quenched disorder is present). Many numerical results exist on these potential energy landscapes, notably for Lennard-Jones and soft-sphere glass forming mixtures 4]. Scaling arguments relating the number of critical points (points where the gradient of the potential energy vanishes) of various types also exist [5] and the onset of the glass transition is often identified with the point where the critical points in the free energy landscape become dominated by minima [6]. In this Letter we compute the average number of critical points of a Gaussian potential, on a high dimensional space, at fixed value of the potential and at fixed number of negative eigenvalues at the critical point.

We shall consider a Gaussian field $\phi$ defined over volume $V$ of an $N$-dimensional Euclidean space. The field has zero mean and correlation function:

$$
\langle\phi(\mathbf{x}) \phi(\mathbf{y})\rangle=N f\left(\frac{(\mathbf{x}-\mathbf{y})^{2}}{2 N}\right) .
$$

This scaling in $N$ ensures the existence of the thermodynamic limit (as $N \rightarrow \infty$ ) of what is known as the toy model, whose statics [7] and dynamics [8] have been studied in the literature. The version of the toy model corresponding to our study here is a single particle in the potential $V(\mathbf{x})=\phi(\mathbf{x})$. In the harmonically confined version of the toy model the potential energy of the particle is $V(\mathbf{x})=\phi(\mathbf{x})+\frac{1}{2} \mu \mathbf{x}^{2}$, and the presence of the confining potential means that the volume $V$ of the system can be taken to infinity. The total number of critical points for the toy model with confining potential was studied by Fyodorov [9] as a function of $\mu$. Here we study the case without confining potential in a finite volume. We give explicit expressions for the average of the number, $\mathcal{N}(\alpha, \epsilon)$, of critical points as a function of index $N \alpha$ and their energy $N \epsilon$ in the form $\langle\mathcal{N}(\alpha, \epsilon)\rangle \sim \exp [N \Sigma(\alpha, \epsilon)]$, where $\Sigma(\epsilon, \alpha)$ is the "complexity", thereby giving a complete picture of the geometry of critical points. Our method can also be applied to the confined case where $\mu \neq 0$ and details will be published elsewhere [10]. For the purposes of this paper we consider the unconfined case as it is more relevant to landscape scenarios in string theory and other translationally invariant systems and also because the results are more explicit.

For an $N \times N$ matrix $H$ the index of $H$, denoted by $\mathcal{I}(H)$, is defined as the number of negative eigenvalues of $H$. The number of critical points of energy $E=N \epsilon$ and index $\alpha N$ is given by the generalized Kac-Rice formula

$$
\begin{aligned}
\mathcal{N}(\alpha, \epsilon)= & \int_{V} d \mathbf{x} \prod_{i=1}^{N} \delta\left(\partial_{i} \phi(\mathbf{x})\right)|\operatorname{det} H(\mathbf{x})| \\
& \delta(\phi(\mathbf{x})-N \epsilon) \delta(\mathcal{I}(H(\mathbf{x}))-N \alpha),
\end{aligned}
$$

where $H(\mathbf{x})$ is the Hessian matrix of the field $\phi$ at the point $\mathbf{x}$, with elements $H_{i j}(\mathbf{x})=\partial_{i} \partial_{j} \phi(\mathbf{x})$. The average of $\mathcal{N}(\alpha, \epsilon)$ is then given, using the spatial translational invariance of the field $\phi$, as

$$
\begin{aligned}
\langle\mathcal{N}(\alpha, \epsilon)\rangle= & V\left\langle\left(\prod_{i=1}^{N} \delta\left(\partial_{i} \phi(\mathbf{0})\right)\right)\right| \operatorname{det}(H(\mathbf{0}) \mid \delta(\phi(\mathbf{0})-N \epsilon)) \\
& \delta(\mathcal{I}(H(\mathbf{0}))-N \alpha)\rangle .
\end{aligned}
$$

The non-vanishing correlation functions for the Gaussian 
random variables $H(\mathbf{0})$ and $\phi(\mathbf{0})$ are:

$$
\begin{aligned}
\langle\phi(\mathbf{0}) \phi(\mathbf{0})\rangle & =N f(0) \\
\left\langle\partial_{i} \partial_{j} \phi(\mathbf{0}) \partial_{k} \partial_{l} \phi(\mathbf{0})\right\rangle & =\frac{f^{\prime \prime}(0)}{N}\left(\delta_{l k} \delta_{i j}+\delta_{l j} \delta_{i k}+\delta_{k j} \delta_{i l}\right) \\
\left\langle\partial_{i} \partial_{j} \phi(\mathbf{0}) \phi(\mathbf{0})\right\rangle & =\delta_{i j} f^{\prime}(0) .
\end{aligned}
$$

Note that positivity conditions for the correlation function of a Gaussian field in Fourier space can be shown to imply [10] $f(0)>0, f^{\prime}(0)<0$ and $f^{\prime \prime}(0)>0$. When $f$ is an analytic function at the origin it is easy to show that the variables $\partial_{i} \phi(\mathbf{0})$ are independent of $H(\mathbf{0})$ and $\phi(\mathbf{0})$, as their correlation with these variables is identically zero. The averaging in Eq. (3) can thus be factorized to give

$$
\begin{aligned}
& \langle\mathcal{N}(\alpha, \epsilon)\rangle=V\left\langle\prod_{i=1}^{N} \delta\left(\partial_{i} \phi(\mathbf{0})\right)\right\rangle \times \\
& \langle\mid \operatorname{det}(H(\mathbf{0}) \mid \delta(\phi(\mathbf{0})-N \epsilon)) \delta(\mathcal{I}(H(\mathbf{0}))-N \alpha)\rangle .
\end{aligned}
$$

Putting this together yields

$$
\langle\mathcal{N}(\alpha, \epsilon)\rangle=\frac{V}{\left(2 \pi\left|f^{\prime}(0)\right|\right)^{\frac{N}{2}}} \Omega(\alpha, \epsilon),
$$

where

$\Omega(\alpha, \epsilon)=\int d \phi \prod_{i \leq j} d H_{i j} p(H, N \epsilon)|\operatorname{det}(H)| \delta(\mathcal{I}(H)-N \alpha)$.

Here $p(H, \phi)$ is the joint probability density of $H$ and $\phi$ (we have dropped the argument $\mathbf{0}$ ). From the correlators in Eq. (4) it can be shown that

$$
p(H, \phi)=C_{N} \exp \left(-N^{2} S_{2}(H)-N S_{1}(H, \phi)\right)
$$

where $C_{N}$ is a normalization constant and the dominant term of the action $S_{2}(H)$ is given by

$$
S_{2}(H)=\frac{1}{4 N f^{\prime \prime}(0)}\left[\operatorname{Tr} H^{2}-\frac{1}{N}(\operatorname{Tr} H)^{2}\right]
$$

and the sub-dominant term $S_{1}(\phi, H)$ is given by

$$
\begin{aligned}
S_{1}(H, \phi)= & \frac{1}{2 f^{\prime \prime}(0) Q}\left[\frac{N+2}{N^{3}} \phi^{2}-\frac{2 f^{\prime}(0)}{N^{2} f^{\prime \prime}(0)} \phi \operatorname{Tr}(H)\right. \\
& \left.+\frac{f(0)}{f^{\prime \prime}(0) N^{2}}(\operatorname{Tr}(H))^{2}\right]
\end{aligned}
$$

where

$$
Q=\left(1+\frac{2}{N}\right) \frac{f(0)}{f^{\prime \prime}(0)}-\frac{f^{\prime}(0)^{2}}{f^{\prime \prime}(0)^{2}}
$$

Positivity conditions for Gaussian correlators can be used to show that $Q>0$ for $N$ finite [10]. However it is possible that $Q^{*}=\lim _{N \rightarrow \infty} Q=\frac{f(0)}{f^{\prime \prime}(0)}-\frac{f^{\prime}(0)^{2}}{f^{\prime \prime}(0)^{2}}$ is zero. A case, often used in simulations of diffusion in random media [11], where this occurs is for $f(x)=\exp \left(-x / l^{2}\right)$, where $l$ is the correlation length. In this case the action $S_{1}$ is order $N$ and some modifications of our approach are needed. For the moment we will proceed by examining the case $Q^{*} \neq 0$. We remark that as the field $\phi$ is statistically isotropic we see that the actions $S_{2}$ and $S_{1}$ are invariant under the action of the rotation group $O(N)$ and that the associated measure thus describes a Gaussian Orthogonal Ensemble (GOE). Notice that if we integrate over the field $\phi$ we find the marginal distribution for $H, p_{m}(H)$, is given by

$$
p_{m}(H)=C_{N}^{\prime} \exp \left(-\frac{N}{4 f^{\prime \prime}(0)}\left[\operatorname{Tr} H^{2}-\frac{1}{N+2}(\operatorname{Tr} H)^{2}\right]\right)
$$

where $C_{N}^{\prime}$ is a normalization constant and recovers the result of Fyodorov 9]. Note that in the above one can take the limit $Q^{*}=0$ without any problems.

We now apply the standard result for the GOE where the integral over $H$ can be reduced to an integral over its eigenvalues [12]:

$$
p(H, \phi) \prod_{i \leq j} d H_{i j}=C_{N}^{\prime \prime} p\left(\lambda_{i}, \phi\right) \prod_{i<j}\left|\lambda_{i}-\lambda_{j}\right| \prod_{i=1}^{N} d \lambda_{i}
$$

where $C_{N}^{\prime \prime}$ is a normalization constant. The key step is now to introduce the normalized density of eigenvalues defined as

$$
\rho(\lambda)=\frac{1}{N} \sum_{i=1}^{N} \delta\left(\lambda-\lambda_{i}\right)
$$

We now adopt the constrained Coulomb gas approach recently introduced in [13 and using standard methods of functional integration [10] we may write

$$
\Omega(\alpha, \epsilon)=\frac{\Theta(\alpha, \epsilon)}{D}
$$

where

$$
\begin{aligned}
& \Theta(\alpha, \epsilon)=\int d[\rho] \exp \left(-N^{2} \mathcal{S}_{2}(\rho)-N \mathcal{S}_{1}(\rho, \epsilon)\right) \\
& \delta\left(\int_{-\infty}^{0} d \lambda \rho(\lambda)-\alpha\right) \delta\left(\int_{-\infty}^{\infty} d \lambda \rho(\lambda)-1\right) .
\end{aligned}
$$

The key point and major simplification in Eq. (16) is that the constraint on the index becomes a simple integral constraint on the functional $\rho$. The effective actions in Eq. (16) are given by

$$
\begin{aligned}
\mathcal{S}_{2}(\rho) & =\frac{1}{4 f^{\prime \prime}(0)}\left[\int d \lambda \lambda^{2} \rho(\lambda)-\left(\int d \lambda \lambda \rho(\lambda)\right)^{2}\right] \\
& -\frac{1}{2} \int d \lambda d \lambda^{\prime} \rho(\lambda) \rho\left(\lambda^{\prime}\right) \ln \left(\left|\lambda-\lambda^{\prime}\right|\right)
\end{aligned}
$$


and

$$
\begin{aligned}
& \mathcal{S}_{1}(\rho, \alpha, \epsilon)=\int d \lambda \rho(\lambda)(\ln (\rho(\lambda))-\ln (|\lambda|)) \\
& +\frac{1}{2 Q f^{\prime \prime}(0)}\left[\frac{N+2}{N} \epsilon^{2}-2 \frac{f^{\prime}(0)}{f^{\prime \prime}(0)} \epsilon \int d \lambda \lambda \rho(\lambda)\right. \\
& \left.+\frac{f(0)}{f^{\prime \prime}(0)}\left(\int d \lambda \lambda \rho(\lambda)\right)^{2}\right]
\end{aligned}
$$

The term $D$ in (15) is a normalization factor given by

$$
D=\int d[\rho] \exp \left(-N^{2} \mathcal{S}_{2}[\rho]-N \mathcal{S}_{1}^{\prime}(\rho)\right)
$$

with

$$
\mathcal{S}_{1}^{\prime}(\rho)=\frac{1}{2 f^{\prime \prime}(0)} \frac{N}{N+2}\left(\int d \lambda \lambda \rho(\lambda)\right)^{2}+\int d \lambda \rho(\lambda) \ln \rho(\lambda) .
$$

The above functional integrals can be evaluated by saddle-point. The order $N^{2}$ action $\mathcal{S}_{2}$ has a zero mode as it is independent of the average of the eigenvalues,

$$
\bar{\lambda}=\int d \lambda \lambda \rho(\lambda)
$$

Note that in the usual Gaussian ensembles the second term of $\mathcal{S}_{2}$ is absent, thus if the average value of the eigenvalues is shifted from zero (and thus we shall see later the value of $\alpha$ is shifted from $1 / 2$ ) then the constraint increases the action $\mathcal{S}_{2}$ by a finite value and thus the probability of finding a Gaussian matrix with $\alpha \neq 1 / 2$ is of order $\exp \left(-N^{2} \theta(\alpha)\right)$ making the probability of minima for instance extremely small [13, 14, 15]. This is not the case for the Hessian of a Gaussian random field at a critical point due to the presence of this zero mode. The degeneracy of the zero mode is however lifted by the order $N$ action. The value of $\rho$ minimizing the action $\mathcal{S}_{2}$ is given by a Wigner semi-circle 16] law up to an undetermined shift (the zero mode)

$$
\rho_{s c}(\lambda)=\frac{1}{2 \pi f^{\prime \prime}(0)}\left(4 f^{\prime \prime}(0)-(\lambda-\bar{\lambda})^{2}\right)^{\frac{1}{2}},
$$

which has support $\lambda \in\left[\bar{\lambda}-2 \sqrt{f^{\prime \prime}(0)}, \bar{\lambda}+2 \sqrt{f^{\prime \prime}(0)}\right]$. This distribution is stiff and any departure from its form will reduce the number of critical points with index fixed to be non-zero by a factor $\exp \left(-N^{2} \theta(\alpha)\right.$ ) (with possible $\log (N)$ corrections to the prefactor $N^{2}$ in the case where $\alpha$ is not strictly equal to one or zero [14]).

Consequently the order $N^{2}$ terms in the saddle-point expressions for $\Omega$ and $D$ cancel as does the order $N$ entropy term $\int d \lambda \rho(\lambda) \ln (\rho(\lambda))$, which is clearly independent of $\bar{\lambda}$. In addition the saddle-point of $D$ is clearly at $\bar{\lambda}=0$. We thus find, that to leading order, the complex- ity of critical points is given by

$$
\begin{aligned}
& \Sigma(\alpha, \epsilon)=\frac{\ln (\mathcal{N}(\alpha, \epsilon))}{N} \\
= & -\frac{1}{2 f^{\prime \prime}(0) Q}\left[\epsilon^{2}-2 \frac{f^{\prime}(0)}{f^{\prime \prime}(0)} \bar{\lambda} \epsilon+\frac{1}{2} P \bar{\lambda}^{2}\right] \\
& +\frac{1}{2} \ln \left(\frac{f^{\prime \prime}(0) L^{2}}{2 \pi e\left|f^{\prime}(0)\right|}\right),
\end{aligned}
$$

where we have made use of the result

$$
\int d \lambda \rho_{s c}(\lambda) \ln (|\lambda|)=\frac{\bar{\lambda}^{2}}{4 f^{\prime \prime}(0)}+\frac{1}{2}\left(-1+\ln \left(f^{\prime \prime}(0)\right)\right),
$$

valid in the regime of interest, $|\bar{\lambda}| \leq 2 \sqrt{f^{\prime \prime}(0)}$, and have defined

$$
P=\frac{f^{\prime}(0)^{2}}{f^{\prime \prime}(0)^{2}}+\frac{f(0)}{f^{\prime \prime}(0)}\left(1-\frac{2}{N}\right) \approx \frac{f^{\prime}(0)^{2}}{f^{\prime \prime}(0)^{2}}+\frac{f(0)}{f^{\prime \prime}(0)} .
$$

In addition, the volume $V$ of the system is written as $V=L^{N}$, with $L$ its characteristic length. The value of $\bar{\lambda}$ is determined from the index constraint

$$
\int_{-\infty}^{0} d \lambda \rho_{s c}(\lambda)=\frac{2}{\pi} \int_{\frac{\lambda}{2 \sqrt{f^{\prime \prime}(0)}}}^{1} d y\left(1-y^{2}\right)^{\frac{1}{2}}=\alpha .
$$

We now discuss the consequences of this general result. If we only constrain the energy of the critical points we find a complexity

$$
\Sigma(\epsilon)=-\frac{\epsilon^{2}}{2 f^{\prime \prime}(0) P}+\frac{1}{2} \ln \left(\frac{f^{\prime \prime}(0) L^{2}}{2 \pi e\left|f^{\prime}(0)\right|}\right)
$$

and the most probable value of $\alpha$ can be computed via

$$
\bar{\lambda}(\epsilon)=2 \frac{f^{\prime}(0) \epsilon}{f^{\prime \prime}(0) P},
$$

and then using the relation Eq. (26). We remark that, as $f^{\prime}(0)<0$, then as $\epsilon$ decreases $\bar{\lambda}$ increases meaning that the most probable index $\alpha$ decreases. Also for $\bar{\lambda}>$ $2 \sqrt{f^{\prime \prime}(0)}$ the value of $\alpha$ freezes at $\alpha=0$, meaning that all the eigenvalues are positive and thus the typical critical points below a critical energy $\epsilon_{c}=\left(f^{\prime \prime}(0)\right)^{\frac{3}{2}} P / f^{\prime}(0)$ are minima. If only the index of the critical point is constrained then we find that

$$
\Sigma(\alpha)=-\frac{\bar{\lambda}^{2}}{4 f^{\prime \prime}(0)}+\frac{1}{2} \ln \left(\frac{f^{\prime \prime}(0) L^{2}}{2 \pi e\left|f^{\prime}(0)\right|}\right)
$$

and that the most probable energy at a given value of $\alpha$ (equivalently $\bar{\lambda}$ ) is

$$
\epsilon(\bar{\lambda})=\frac{f^{\prime}(0)}{f^{\prime \prime}(0)} \bar{\lambda}
$$

From this we see that the most probable energy of minima, $\epsilon_{m}$, is $\epsilon_{m}=2 f^{\prime}(0) / \sqrt{f^{\prime \prime}(0)}$. In fact one can show 
that $\epsilon_{m} \geq \epsilon_{c}$ with equality in the case $Q^{*}=0$ [10]. This means that generically most minima are at the energy $\epsilon_{m}$ but it is only below the energy $\epsilon_{c}$ that they are the dominant critical points. For $\bar{\lambda}>2 \sqrt{f^{\prime \prime}(0)}$ all critical points are minima, so the complexity of minima is

$$
\Sigma_{\min }=-1+\frac{1}{2} \ln \left(\frac{f^{\prime \prime}(0) L^{2}}{2 \pi e\left|f^{\prime}(0)\right|}\right) .
$$

Thus there is a characteristic length $L_{c}=$ $\sqrt{2 \pi e^{3}\left|f^{\prime}(0)\right| / f^{\prime \prime}(0)}$ below which there are almost surely no minima.

We now discuss the case where $Q^{*}=0$. In this case the first line of Eq. (18) is of order one but the other terms are of order $N$. We see, however, that the order $N$ terms only depend on $\rho$ via $\bar{\lambda}$. This means that at the saddle-point $\rho$ still has the Wigner form of Eq (22). Consequently we find that

$$
\begin{aligned}
\Sigma(\alpha, \epsilon)= & -\frac{N}{4 f(0)}\left[\frac{N+2}{N} \epsilon^{2}-2 \frac{f^{\prime}(0)}{f^{\prime \prime}(0)} \bar{\lambda} \epsilon+\frac{1}{2} P \bar{\lambda}^{2}\right] \\
& +\frac{1}{2} \ln \left(\frac{f^{\prime \prime}(0) L^{2}}{2 \pi e\left|f^{\prime}(0)\right|}\right),
\end{aligned}
$$

where we have kept the $N$-dependent prefactor of $\epsilon^{2}$. Completing the square in $\epsilon$, the above result may be written to leading order for large $N$ as

$$
\begin{aligned}
\Sigma(\alpha, \epsilon) & =-\frac{N}{4 f(0)}\left(\epsilon-\frac{f^{\prime}(0)}{f^{\prime \prime}(0)} \bar{\lambda}\right)^{2}-\frac{\bar{\lambda}^{2}}{4 f^{\prime \prime}(0)} \\
& +\frac{1}{2} \ln \left(\frac{f^{\prime \prime}(0) L^{2}}{2 \pi e\left|f^{\prime}(0)\right|}\right),
\end{aligned}
$$

which means that when the index is fixed (i.e. $\bar{\lambda}$ is fixed) then the critical points of index given by $\bar{\lambda}$ are concentrated about the energy $\epsilon=\bar{\lambda} f^{\prime}(0) / f^{\prime \prime}(0)$ as before but the fluctuations about this energy are $O(1 / N)$ as opposed to fluctuations of $O(1 / \sqrt{N})$ in the case where $Q^{*} \neq 0$. The same scaling holds for the fluctuations about the most probable index, given by Eq. (28), when the energy is fixed.

A study of the p-spin spherical spin glass model [17] has shown that at energies below a threshold energy almost all critical points are minima. However, at the threshold energy all indices are equally probable. In the model studied here there is a similar threshold energy below which almost all critical points are minima. However, as this energy is increased the index is concentrated around a well defined value up to an upper threshold energy beyond which all critical points are maxima.

Numerical investigations of the critical points of glass formers [4] are carried out by equilibrating the system, freezing it and then finding nearby critical points. In most of these studies a clear linear relation is found between the index $\alpha$ and the potential energy $\epsilon$ for values of $\alpha$ between 0 and about 0.1 . This is in contrast to the results found here where a linear relation exists between $\bar{\lambda}$ and $\epsilon$, and the relation between $\alpha$ and $\epsilon$ must be found via Eq. (26). For small $\alpha$ we find the relation

$$
\alpha \approx \frac{4 \sqrt{2}}{3 \pi}\left|\frac{\epsilon-\epsilon_{c}}{\epsilon_{c}}\right|^{\frac{3}{2}}
$$

This difference may be due to the fact that the Gaussian landscape is quite different from that of glass formers. However, it may also be that the preparation of the system in an equilibrium configuration, before starting the critical point search, biases the statistics of the critical points found, whereas our calculation is based on a purely flat measure.

In summary, we have provided a complete description of the distribution of critical points of Gaussian fields in high-dimensional spaces, as a function of their energy and index. The generalization to the case of a confining harmonic potential will be presented elsewhere [10]. Finally the analytic results presented here will also be useful to test numerical methods used to explore the critical points of potentials on large-dimensional spaces.

We thank Satya Majumdar for useful discussions. AB thanks Université Paul Sabatier, Toulouse, for its hospitality while this work was being done.

[1] R.A. Adler, The geometry of random fields, J. Wiley and Sons, New York, (1981).

[2] L. Susskind, arXiv:hep-th/0302219 M.R. Douglas, B. Shiffman, and S. Zelditch, Commu. Math. Phys. 252, 325 (2004).

[3] D.J. Wales, Energy landscapes:with applications to clusters biomolecules and glasses (Cambridge University Press) (2004).

[4] K. Broderix, K.K. Battacharya, A. Cavagna, A. Zippelius and I. Giardina, Phys. Rev. Lett. 85, 5360 (2000); T.S. Grigera, A. Cavagna, I. Giardina and G. Parisi, Phys. Rev. Lett. 88055502 (2002); J.P.K Doyle and D.J. Wales, J. Chem. Phys. 116, 3777 (2002); P. Sha and C. Chakravaty, J. Chem. Phys. 115, 8784 (2001).

[5] D.J. Wales and J.P.K Doyle, J. Chem. Phys. 119, 12409 (2003);M.S. Shell, P.G. Debenedetti and A.Z. Panagiotopoulos, Phys. Rev. Lett. 92, 035506 (2004).

[6] J. Kurchan, L. Laloux, J. Phys. A 29, 1929 (1996); S. Franz and M. A. Virasoro, J. Phys. A 33, 891 (2000);A. Cavagna, Europhys. Lett. 53, 490 (2001).

[7] M. Mézard and G. Parisi, J. Phys. I France 1, 809 (1991); A. Engel, Nucl. Phys. B 410, 617 (1993).

[8] H. Kinzelbach and H. Horner, J. Phys. I 31329 (1993); S. Franz and M. Mézard, Physica A 210, 48 (1994); L.F. Cugliandolo and P. Le Doussal, Phys. Rev. E 531525 (1996).

[9] Y.V. Fyodorov Phys. Rev. Lett.92, 240601 (2004) ; ibid Acta Physica Polonica B, 36, 2699 (2005).

[10] A.J. Bray and D.S. Dean, in preparation.

[11] D.S. Dean, I.T. Drummond and R.R. Horgan, J. Phys. A 27, 5135 (1994). 
[12] M.L. Mehta, Random Matrices, 2nd Edition, (Academic Press) (1991).

[13] D.S. Dean and S.N.Majumdar, Phys. Rev. Lett. 97, 160201 (2006)

[14] A. Cavagna, J.P. Garrahan and I,. Giardina, Phys. Rev. B 61, 3960 (2000).

[15] A. Aazami and R. Easther, J. Cosmol. Astropart. Phys.
JCAP03 013 (2006).

[16] E.P. Wigner, Proc. Cambridge Philos. Soc. 47, 790 (1951).

[17] A. Cavagna, I. Giardina and G. Parisi, Phys. Rev. B 57, 11251 (1998). 